\title{
Single-stranded heteroduplex intermediates in $\lambda$ Red homologous recombination
}

\author{
Marcello Maresca ${ }^{1}$, Axel Erler ${ }^{1}$, Jun Fu ${ }^{1}$, Anne Friedrich ${ }^{1}$, Youming Zhang ${ }^{2}$, A Francis Stewart ${ }^{*}$
}

\begin{abstract}
Background: The Red proteins of lambda phage mediate probably the simplest and most efficient homologous recombination reactions yet described. However the mechanism of dsDNA recombination remains undefined.

Results: Here we show that the Red proteins can act via full length single stranded intermediates to establish single stranded heteroduplexes at the replication fork. We created asymmetrically digestible dsDNA substrates by exploiting the fact that Reda exonuclease activity requires a 5' phosphorylated end, or is blocked by phosphothioates. Using these substrates, we found that the most efficient configuration for dsDNA recombination occurred when the strand that can prime Okazaki-like synthesis contained both homology regions on the same ssDNA molecule. Furthermore, we show that Red recombination requires replication of the target molecule.

Conclusions: Hence we propose a new model for dsDNA recombination, termed 'beta' recombination, based on the formation of ssDNA heteroduplexes at the replication fork. Implications of the model were tested using (i) an in situ assay for recombination, which showed that recombination generated mixed wild type and recombinant colonies; and (ii) the predicted asymmetries of the homology arms, which showed that recombination is more sensitive to non-homologies attached to $5^{\prime}$ than $3^{\prime}$ ends. Whereas beta recombination can generate deletions in target BACs of at least $50 \mathrm{~kb}$ at about the same efficiency as small deletions, the converse event of insertion is very sensitive to increasing size. Insertions up to $3 \mathrm{~kb}$ are most efficiently achieved using beta recombination, however at greater sizes, an alternative Red-mediated mechanism(s) appears to be equally efficient. These findings define a new intermediate in homologous recombination, which also has practical implications for recombineering with the Red proteins.
\end{abstract}

\section{Background}

Homologous recombination (HR) is central to all replicating cells because it is a main pathway for the rescue of replication fork catastrophes $[1,2]$. When a replication fork encounters a single-strand nick or unrepaired DNA damage, a double-strand break is generated and the replication fork collapses. HR serves to reset the replication fork through a process involving a 5 ' to 3 ' exonuclease or helicase, which generates a 3'-ended singlestranded region from the double-strand break. Singlestrand binding proteins then promote joint molecule formation by hybridization between the single-stranded region and its recently replicated complementary sequence. Once the complementary sequence is found, the replication fork can be restarted.

\footnotetext{
* Correspondence: stewart@biotec.tu-dresden.de
${ }^{1}$ Genomics, Technische Universität Dresden, BiolnnovationsZentrum, Tatzberg

* Correspondence: stewart@biotec.tu-dresden.de
${ }^{1}$ Genomics, Technische Universität Dresden, BiolnnovationsZentrum, Tatzberg 47-51, D-01307 Dresden, Germany
}

Single-strand binding proteins involved in HR promote the formation of joint molecules in two different ways, either strand invasion or annealing. Strand invasion requires a RecA/RAD51 superfamily member to mediate the search for complementarity between the singlestranded DNA (ssDNA) region and double-stranded DNA (dsDNA) through a triple-stranded intermediate termed a displacement loop (D-loop). Annealing is mediated by single-strand annealing proteins (SSAPs) between complementary ssDNAs [3]. By sequence alignment, three families of SSAPs have been defined based on RAD52, RecT/Red $\beta$ and Erf [4]. Recently we discovered that the RAD52 and $\operatorname{RecT} / \operatorname{Red} \beta$ families are related [5], suggesting that annealing mechanisms related to HR may share a common basis.

$\mathrm{HR}$ is also central to the technologies of gene targeting and recombineering. Gene targeting is the process whereby the genome of a living cell is altered by the 
introduction of recombinant DNA into a specific site, mediated by the endogenous HR machinery [6-9]. Recombineering mainly refers to the engineering of recombinant DNA by HR [7,10-12]. Both gene targeting and recombineering usually involve the generation of a linear dsDNA copy of a segment of the chosen target DNA. A stretch of heterologous dsDNA, which usually contains a selectable gene, is introduced into the middle of the copy. The incorporation of the linear dsDNA by HR incorporates the central heterologous sequence and so alters the target by 'replacement'. This application of HR differs from endogenous HR because it does not involve the repair of a DSB, rather it is stimulated by the two DSB-like ends of the linear copy.

The standard model for replacement targeting by HR involves $5^{\prime}$ to $3^{\prime}$ resection at both ends (Figure 1A), followed by a double crossover [13-16]. However some evidence indicates that other explanations may be valid. Two alternatives to the standard model were discussed by Paques and Haber based on observations made in S. cerevisiae [15]. They favoured the evidence of Morrow et al [17] for a double primed replication event that has similarities to break induced replication (BIR), leading to extensive chromosomal duplication. They also mentioned the data of Leung et al [18] who proposed a model based on the assimilation of a solo singlestranded intermediate.

Here we focus on recombination mediated by the Red proteins of $\lambda$ phage. The Red operon includes a 5 ' to $3^{\prime}$ exonuclease, Red $\alpha$ [19-21], an SSAP, $\operatorname{Red} \beta$ [5,22,23] and $\operatorname{Red} \gamma$, which is an inhibitor of the major $E$. coli exonuclease, RecBCD [24,25]. Whereas the role for Red $\alpha$ to generate ssDNA regions from dsDNA ends is clear, questions regarding the ability of $\operatorname{Red} \beta$ to mediate strand invasion have been raised [3,26,27]. Kuzminov $[3,28]$ argued convincingly that $\operatorname{Red} \beta$ can only mediate annealing. However the functional discovery of apparent $\operatorname{Red} \beta$ strand invasion activity in the absence of RecA [29,30] presented a conundrum. Ellis et al [31] provided part of the solution. They observed a strand specific bias in site-directed mutagenesis mediated by Red $\beta$. Singlestranded oligonucleotides that could serve as primers for Okazaki fragment synthesis were more efficient than their complementary oligonucleotides. Hence the model was proposed that $\operatorname{Red} \beta$ anneals oligonucleotides into single-stranded regions at the replication fork $[7,31,32]$. This model gained further support from the observation that removal of the mismatch repair pathway enhanced oligonucleotide-directed mutagenesis [33,34].

Whereas this model explains oligonucleotide-directed $\mathrm{HR}$, the mechanism of Red-mediated dsDNA HR remains unexplained. The standard model for replacement HR has been favoured $[7,35,36]$. However this double crossover model encounters a problem if $\operatorname{Red} \beta$

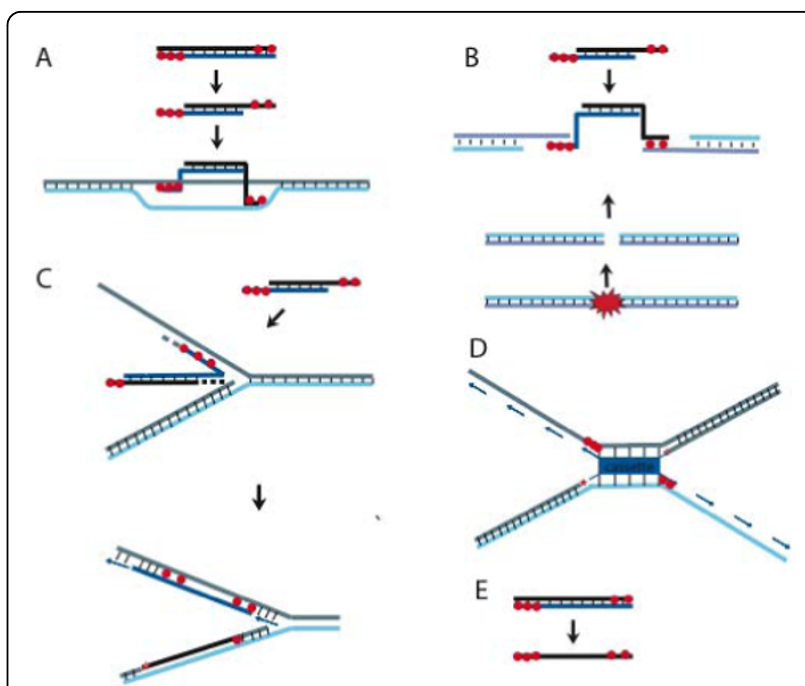

Figure 1 Proposed recombination models based on a linear dsDNA substrate. (A) Strand invasion model. A linear DNA is resected on both ends by a $5^{\prime}$ to $3^{\prime}$ exonuclease (e.g. Reda, RecE, not shown) and the single stranded $3^{\prime}$ tails are bound by a recombinase (Red $\beta$ or RecT; red circles) to generate nucleoprotein filaments on each end of the linear DNA, which promote a doublecrossover joint molecule by strand invasion into target dsDNA. (B) DNA annealing model. Exonucleolytic resection and recombinase assembly is the same as A. However, the nucleoprotein filaments anneal directly to complementary single stranded 3 ' tails generated from a DSB in the target DNA molecule. (C) Chicken foot model. Exonucleolytic resection and recombinase assembly is the same as A. One end of the linear DNA anneals to a single-stranded region at the lagging strand of the replication fork. A 'chicken foot' intermediate is formed. Backtracking of the chicken foot intermediate by branch migration reestablishes the replication fork (adapted from [7]). D) Bridge model. The linear DNA is inserted in between two colliding replication forks. Each nucleoprotein filament at the ends of the cassette (shown in blue) anneals to the lagging strand of one or the other replication fork thereby inserting the cassette in the middle of the two replication forks (adapted from [7]). E) Schematic illustration of an alternative digestion product after $5^{\prime}$ to $3^{\prime}$ Red $\alpha$ exonuclease activity. Instead of degradation from both ends of the linear dsDNA, Reda starts only at one end and complete digestion leaves an intact single-strand behind.

cannot catalyze strand invasion. Hence the mechanism illustrated in the diagram of Figure $1 \mathrm{~A}$ is not possible without modification. An alternative to the standard model invokes the occurrence of an adventitious DSB near the chosen targeting site (Figure 1B). This is possible but the high HR efficiencies achieved using Red recombination cast doubt on this presumption. Accordingly, Court et al [7] proposed two further models based on either a chicken foot intermediate (Figure 1C) or the establishment of a bidirectional replication fork (Figure 1D). Neither of these two models is entirely satisfactory. The chicken foot intermediate, as part of the RecG pathway [37], serves to pass damaged nucleotides. However Red recombination can insert thousands of base 
pairs of heterologous sequence and removal of RecG increases Red recombination efficiencies, indicating that RecG ejects recombination intermediates [38]. The bidirectional replication fork may be reminiscent of BIR, but is without support from evidence. More recently, a template-switching model based on invasion of the replication fork by the 3 ' end of an ssDNA/dsDNA product of Red $\alpha$ exonuclease digestion has been proposed [39]. However this model cannot explain replacement HR without some modification or extension.

An accidental discovery led us to reconsider the conundrum of Red-mediated dsDNA HR. Inadvertently we noticed a significant number of very late growing colonies produced by a boiled dsDNA control. Examination of these colonies revealed that they all contained the intended recombination (data not shown). Although we later found that boiled dsDNA is very inefficient compared to optimal recombineering practice, this unexpected observation led us to re-evaluate our presumptions and consider a new model for replacement HR by Red (Figure 1E). Rather than beginning with symmetrical resection of the linear substrate by Red $\alpha$, here we consider the idea that Red $\alpha$ eliminates one strand entirely leaving the other intact. Then $\operatorname{Red} \beta$ mediates the incorporation of the intact strand into the replication fork to effect replacement HR.

\section{Results}

Most recombination experiments described here employed linear substrates that included $50 \mathrm{nt} / \mathrm{bp}$ of continuous sequence identity to the target molecules at each end (termed the 'homology arms'), flanking a gene encoding resistance to an antibiotic. The target molecules were intact circles, being either plasmids, Bacterial Artificial Chromosomes (BACs) or the E. coli chromosome. Recombination was scored as colonies that acquired resistance to the corresponding antibiotic under conditions where all antibiotic resistant colonies were due to the intended recombination, as evaluated by DNA analyses (data not shown). The recombination reactions were carried out with our standard protocol, which has been optimized for convenience and productivity. All experiments were performed in $r e c A$ strains.

\section{Replication is required for Red recombination with dsDNA substrates}

In the absence of RecA, dsDNA recombination by the Red proteins requires replication, however the mechanism remains undefined [39]. To define the step at which replication is required for Red recombination with dsDNA substrates, we performed the experiment shown in Figure 2A. We used an R6K plasmid, which requires the Pi protein to replicate $[40,41]$, as well as a linear dsDNA fragment that included a colE1 origin and the blasticidin resistance gene. The homology arms were chosen so that recombination would replace the R6K origin with the colE1 origin. Thereby the recombination product will have a functioning colE1 replication origin. If replication is required to initiate recombination, then the Pi protein must be present so that the R6K plasmid is replicating. On the other hand, if replication is only required to amplify the initiating recombination complex, then the insertion of the colE1 origin should suffice.

The two DNAs were co-electroporated into an E. coli strain that contained Red $\alpha \beta \gamma$ with or without expression of Pi (strains GB2005 and GB2005-pir respectively). Recombination only occurred in the presence of $\mathrm{Pi}$, demonstrating a need for replication of the R6K plasmid before recombination (Figure 2B). To support this result, we altered the experiment to direct recombination towards the pir gene (Figure 2C). Rather than exchange the origin of replication, here recombination will restore expression of $\mathrm{Pi}$, which will permit replication of the plasmid. However no plasmid replication occurred without supplying $\mathrm{Pi}$ in trans at the start of the experiment (Figure 2D).

These results show that the target molecule must be replicating to permit the initiation of dsDNA recombination.

Rethinking the dsDNA intermediate in Red recombination Previous ideas about Red recombination with dsDNA substrates have assumed that the reaction initiates with action by Red $\alpha$ exonuclease to create a symmetrically resected ssDNA/dsDNA intermediate (as illustrated in Figure 1A) that then hybridizes to form some kind of joint molecule. To challenge this assumption and test the possibility that the recombination intermediate is a full-length ssDNA, we exploited the fact that Red $\alpha$ prefers to begin exonuclease activity on a 5 ' phosphorylated rather than hydroxylated (dephosphorylated) end [21,42]. A dsDNA substrate was prepared carrying either phosphorylated $(\mathrm{P})$ or hydroxylated $(\mathrm{O}) 5^{\prime}$ ends in all four combinations (OO, OP, PO, PP) as illustrated in Figure 3. These substrates were digested with Red $\alpha$ in vitro and the products analyzed by single-strand conformation polymorphism (SSCP), which can separate complementary single-strands by gel electrophoresis $[43,44]$. As can be seen in Figure 3A, Red $\alpha$ digested only the phosphorylated strand in the OP and PO substrates, whereas the OO substrate was not digested on either strand and the PP substrate was digested on both, producing some different ssDNA products.

Subsequently we used these OP and PO ssDNA products in a recombination reaction to insert the blasticidin $(b s d)$ resistance gene into a BAC (Figure 3B). Because $\operatorname{Red} \alpha$ is not required for $\operatorname{Red} \beta$ mediated 

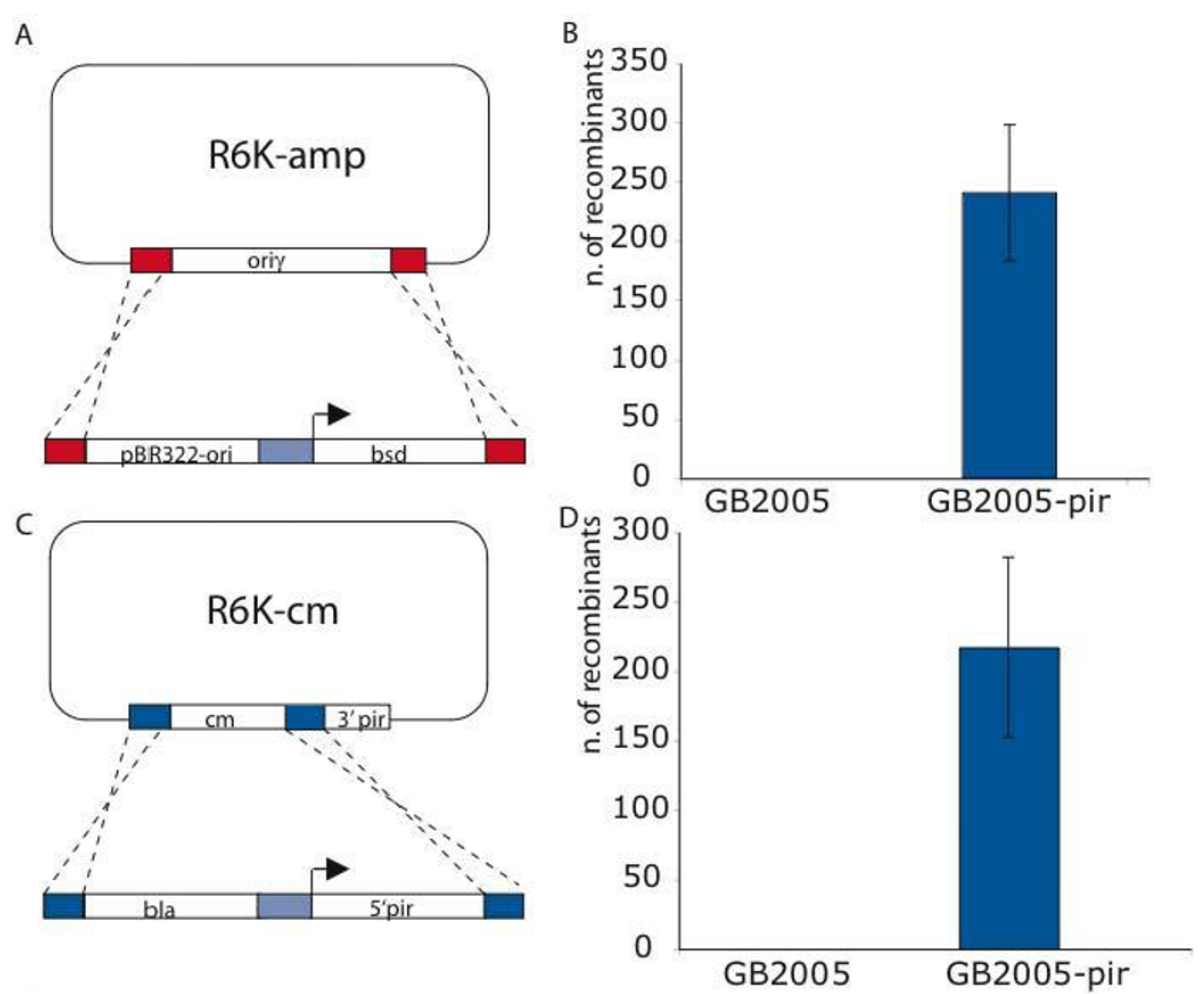

Figure 2 Replication is required for Red recombination. (A) Schematic illustration of an experiment to replace the oriy replication origin in an R6K-amp plasmid with the pBR322 replication origin and the bsd gene for blasticidin resistance. (B) Quantification of recombinant colonies from the experiment shown in (A). Recombination only occurred when the pir gene was expressed in trans from the E. coli chromosome (strain GB2005-pir). No recombination product was found in the absence of pir (strain GB2005). (C) Schematic illustration of an experiment to rebuild the pir gene in an R6K plasmid using an ampicillin resistance gene bla and a $5^{\prime}$ fragment of the pir gene, which completes the pir gene when fused with the 3' end of the pir gene. (D) Quantification of recombinant colonies of the experiment depicted in (C). Similar to the result shown in (B), recombination only occurred when the Pi protein was expressed in trans.

recombination with single-stranded oligonucleotides $[31,32,45]$, the host cells contained only Red $\beta$. In parallel as a positive control, we performed a recombination reaction with single-stranded oligonucleotides to repair a mutation in the kanamycin resistance gene (neo) and so restore kanamycin resistance. As expected from previous observations, the oligonucleotide that can serve as a primer for Okazaki-fragment synthesis $(\mathrm{Lg})$ produced more recombinants than the complementary oligonucleotide (Ld; Figure 3C). The same effect was observed with the ssDNA substrates. That is, the ssDNA that can prime Okazaki-fragment synthesis (low) delivered many more recombinants than the complementary strand. Notably, the two assays could be distinguished by mutations in the mismatch repair pathway (mutS), indicating the two reactions are not identical. As shown before [33,34], mutations in the mismatch repair pathway enhanced oligonucleotide-directed mutagenesis and the enhancement was greater for the leading strand oligonucleotide (Ld). However, we found no mutS effect on ssDNA recombination (Figure $3 \mathrm{C}$ ). Conversely, mutating the 3 ' to 5 ' exonuclease I, $s b c B$ [46,47], significantly enhanced both oligonucleotide and ssDNA directed recombination indicating that both assays are similarly sensitive to 3 ' exposed ssDNA ends.

\section{Testing the ssDNA intermediate in vivo}

To determine whether dsDNA recombination can be processed through a full-length ssDNA intermediate in vivo, we employed the four linear dsDNA substrates (OO, OP, PO, PP) in a recombination assay in the presence of Red $\alpha$ as well as $\operatorname{Red} \beta$ (and $\operatorname{Red} \gamma$ ). The experimental design is shown in Figure 4A. Recombination was directed to either a BAC or the E. coli chromosome. In both cases, the target was established in both orientations (bla and inv-bla), which alters the recombination reaction with respect to the direction of replication. Using an asymmetric substrate (OP), SSCP revealed that Red $\alpha$ rapidly generated the ssDNA intermediate in vivo (Figure 4B). Recombination was most efficient with the asymmetrically phosphorylated substrate whose hydroxylated 5 strand could prime lagging strand synthesis, 

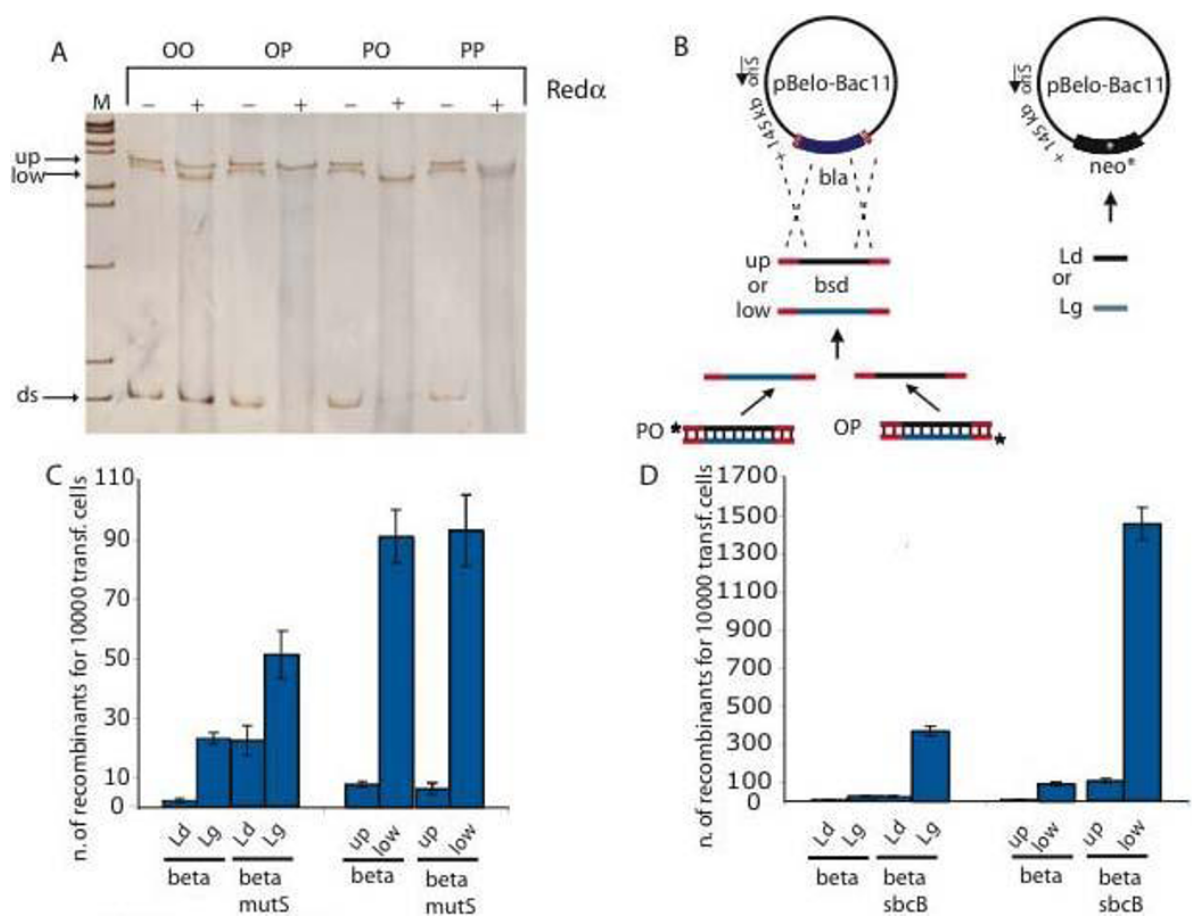

Figure 3 Recombination and oligonucleotide-mediated mutagenesis using ssDNAs. (A) SSCP-PAGE gel of phosphorylated (P) and hydroxylated (O) recombination cassettes in all four combinations (OO, OP, PO, PP) with (+) or without (-) Reda in vitro digestion. The lower bands show undenatured dsDNA (ds) and the upper bands show the slower migrating secondary structures of the single-strands after heat denaturation (up and low). M - size markers. (B) Schematic representations of the recombination reactions, which either replaced the ampicillin

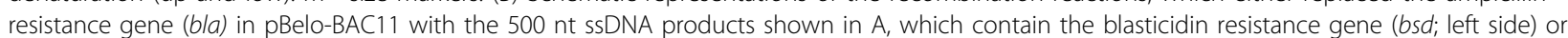
repaired a $4 \mathrm{nt}$ mutation in the kanamycin resistance gene (neo) using $100 \mathrm{nt}$ single-stranded oligonucleotides (right side). Only Red $\beta$ was expressed in these experiments. The oligonucleotides were either complementary to the leading $(\mathrm{Ld})$ or lagging $(\mathrm{Lg}) \mathrm{strands}$. $(\mathrm{C}, \mathrm{D})$

Quantification of recombinant colonies using the experimental designs shown in (B) and comparison of the wild type (wt) to either mutS (C) or $s b c B(D)$ strains.

henceforth referred to as the 'LSP' for lagging strand primer. Inverting the target inverted the strand preference, demonstrating that the PO and OP substrates were both proficient. The same conclusions could be drawn from both the BAC and chromosomal assays (Figure 4C, D). Notably, the next best substrate in all configurations was the doubly hydroxylated one (OO) and in this case inverting the target had no effect.

To challenge these findings in an additional way, we made substrates with phosphothioated bonds at the $5^{\prime}$ ends. We first established that two consecutive phosphothioate bonds is optimal and superior to hydroxylated ends (Figure 4E). Hence we generated dsDNA substrates with two phosphothioated bonds at the $5^{\prime}$ end (S) in combination with phosphorylated (P) or hydroxylated (O) $5^{\prime}$ ends. Again, the most efficient SP orientation had the phosphothioate at the $5^{\prime}$ end of the LSP (Figure 4D). Consistent with predictions, the asymmetrically phosphothioated/phosphorylated (SP, PS) LSPs were more efficient than the hydroxylated/phosphorylated (OP, PO) or phosphothioated/hydroxylated LSPs. Furthermore permutations of $\mathrm{S}$ and $\mathrm{O}$, like the $\mathrm{OO}$ substrate, also delivered good recombination efficiencies. The SO substrates displayed a limited strand preference, whereas the SS substrate showed none.

All of these results are consistent with the following conclusions; (i) 5' phosphothioates or 5 ' end hydroxylation enhanced recombination of the LSP because they convey resistance to $5^{\prime}-3^{\prime}$ exonuclease activity; (ii) this enhancement is amplified when the $5^{\prime}$ end of the other strand is phosphorylated because rapid degradation of the unfavourable strand increases the yield of fulllength, single-stranded, LSP; (iii) when both 5 ' ends are blocked to the exonculease, it is possible that another activity such as a helicase separates the strands so that the full-length LSP can initiate recombination.

As a further test, we designed an experiment to evaluate the recombination efficiencies of symmetrically resected dsDNA intermediates. A pair of phosphothioates were symmetrically placed at increasing distances from the $5^{\prime}$ ends of a substrate as illustrated in Figure 5. That is, the phosphothioates were either $10,20,30,40$, 50,60 or $70 \mathrm{nt}$ from each $5^{\prime}$ end. Both $5^{\prime}$ ends were phosphorylated to promote Red $\alpha$ exonuclease activity. 

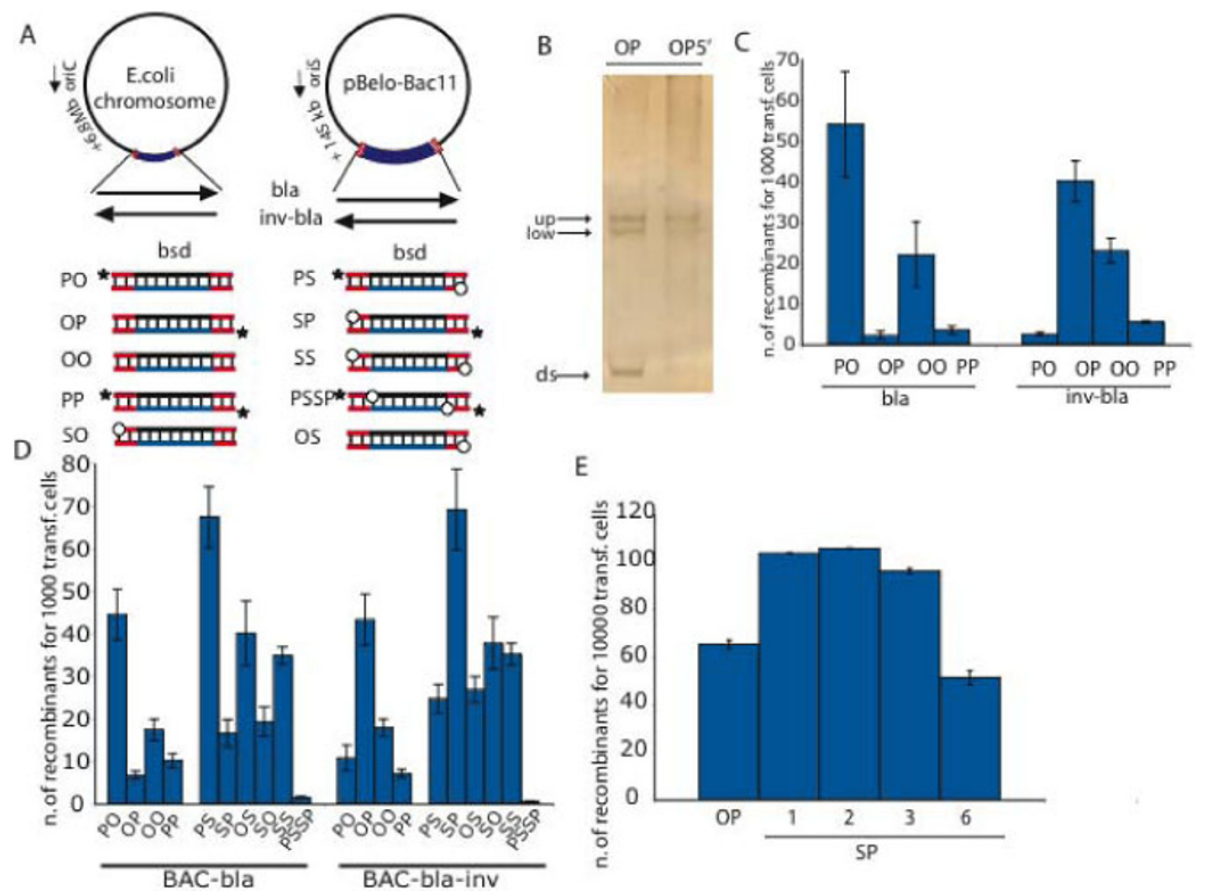

E

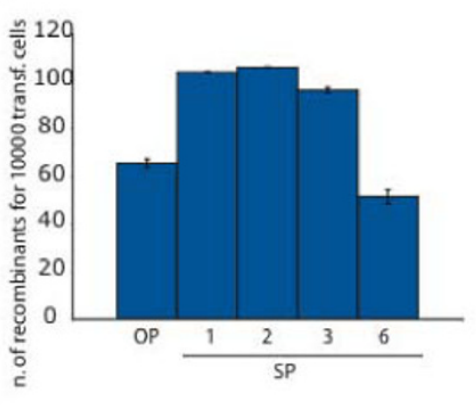

Figure 4 In vivo evidence for the ssDNA intermediate. (A) Schematic representations of recombination reactions to replace the ampicillin (bla) with the blasticidin (bsd) resistance gene in either the $E$. coli chromosome or pBelo-Bac11. The bla gene was present in both orientations (bla, inv-bla) to alter its orientation to the direction of replication, which is indicated by an arrow beside the origin, either oriC or oriS. The bsd gene was PCR amplified to possess different $5^{\prime}$ ends as indicated, either phosphorylated ( $\mathrm{P}$, black stars), hydroxylated (O, no symbol) or phosphothioated (S, open circles) in various combinations. PSSP indicates that both $5^{\prime}$ ends were phosphorylated and the first two backbone linkages were also phosphothioated. All three Red proteins $(\alpha, \beta, \gamma)$ were expressed in these experiments. (B) SSCP-PAGE gel of a hydroxylated/ phosphorylated linear dsDNA substrate (OP) after transformation in vivo. The lower band represents dsDNA (ds) and the upper bands represent the slower migrating secondary structures of the single-strands after heat denaturation (up and low). DNA was harvested from cells immediately after electroporation (OP) or after incubation for 5 minutes at $37^{\circ} \mathrm{C}\left(\mathrm{OP} 5^{\prime}\right)$. (C, D) Quantification of recombinant colonies using the experimental design shown in (A). The targeted loci were either located on the E. coli chromosome (C) or on the pBelo-Bac11 BAC (D) in direct (bla) or inverted (inv-bla) orientation. (E) Kanamycin-resistant colonies after repair of pBelo-Bac11-neo* BAC using dsDNA with zero (OP), one (SP-1), two (SP-2), four (SP-4) or six (SP-6) consecutive phosphothioated bonds at one $5^{\prime}$ end. The other $5^{\prime}$ end was phosphorylated (P).

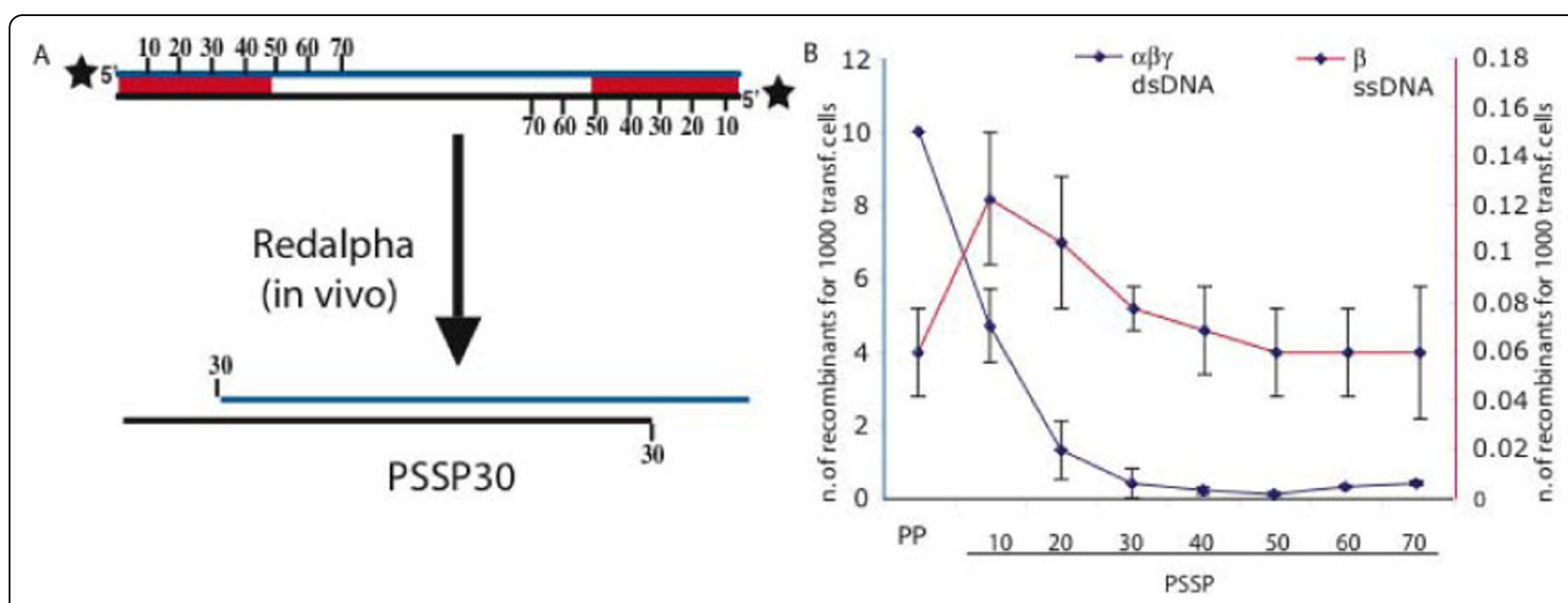

Figure 5 Red recombination of symmetrically resected dsDNA substrates. (A) Schematic representation of linear PSSP dsDNA substrates showing the distance (in nt) to two phosphothioate bonds that were symmetrically positioned from each $5^{\prime}$ end. Additionally, phosphates were present at both 5'ends (black stars). Below the PSSP30 substrate is illustrated after Red $\alpha$ digestion exposing 30 nt ssDNA tails at each end; (B) Recombination efficiencies of the PSSP substrates. Either Red $\alpha$, Red $\beta$ and Red $\gamma$ (blue line, left ordinate) or only Red $\beta$ (red line, right ordinate) were expressed. In the second case (only Red $\beta$ ) the dsDNA substrates were heat denatured prior to electroporation. 
We reasoned that Red $\alpha$ would resect both ends until reaching the phosphothioate bonds, thereby generating the symmetrically resected substrates that were previously believed to be the optimal recombination intermediates. By this previous logic, the series of phosphothiolated substrates up to $70 \mathrm{nt}$ was chosen to include the presumptive best intermediates that have both $50 \mathrm{nt}$ homology arms exposed as ssDNA and the region in between as double-stranded. However, these substrates were very inefficient (Figure 5B), particularly when compared to the SS substrate (Figure 4D). To control for the quality of the substrates, they were boiled to separate the strands and used in a recombination assay mediated by $\operatorname{Red} \beta$ only. Although this $\operatorname{Red} \beta-$ only recombination reaction is very inefficient, each of the internally phosphothioated substrates were equivalent for recombination after boiling demonstrating their integrity for recombination.

Taken together, this evidence supports a new model for Red replacement HR mediated through a singlestrand that forms a heteroduplex at the replication fork, as illustrated in Figure 6. We call this model 'beta' recombination for convenience. To challenge the model, we examined several implications.

\section{Evidence for a heteroduplex intermediate in Red replacement $\mathrm{HR}$}

The beta recombination model predicts that the recombination intermediate is initially a heteroduplex on the lagging strand of both mutant and parental strands. If this heteroduplex is replicated, the product should be one daughter that is parental and one that is mutant. To look for this possibility, we developed an in situ recombination assay based on colony colour. On MacConkey agar, cells carrying an intact malK gene give a red colour whereas mutants are white [48]. The assay was performed in two directions, one to mutate the malK gene and the other to restore it. After brief incubation to permit recombination, cells were plated out at single cell dilution and cultured until the colony colour was apparent (Figure 7). Because an E. coli cell can harbour two or more copies of any gene (after it has been replicated, before cell division), we performed these experiments as a time course of brief incubation before plating. As presented in Table 1, almost all recombination events at the earliest time point resulted in two-colour colonies, indicating that recombination produced a heteroduplex as predicted. Upon longer incubation times, the proportion of single colour colonies increased, which indicated an increasing proportion of colonies formed by cell division before plating as expected.

\section{Asymmetry of the homology arms}

The beta recombination model implies that the homology arms are not equivalent. That is, the homology arm at the 3 ' end of the LSP should anneal first, followed by annealing of the 5' LSP homology arm. Also, the homology arms on the other strand should be unimportant. These asymmetries permit certain tests.

First, we tested the impact of adding non-homologous sequence to the $3^{\prime}$ or $5^{\prime}$ ends of the LSP (Figure $8 \mathrm{~A}$ ). When the non-homology was present on the $5^{\prime}$ end of the LSP (OP5'), recombination was impaired more than when it was on the $3^{\prime}$ end (OP3'). This suggests that additional non-homologous sequences on the 3 ' end, which will impede priming for Okazaki fragment DNA synthesis, are easily removed. Whereas additional nonhomologous sequences at the 5 ' end are a more substantial impediment, possibly because they interfere with the ligation step required to complete the mutant strand.
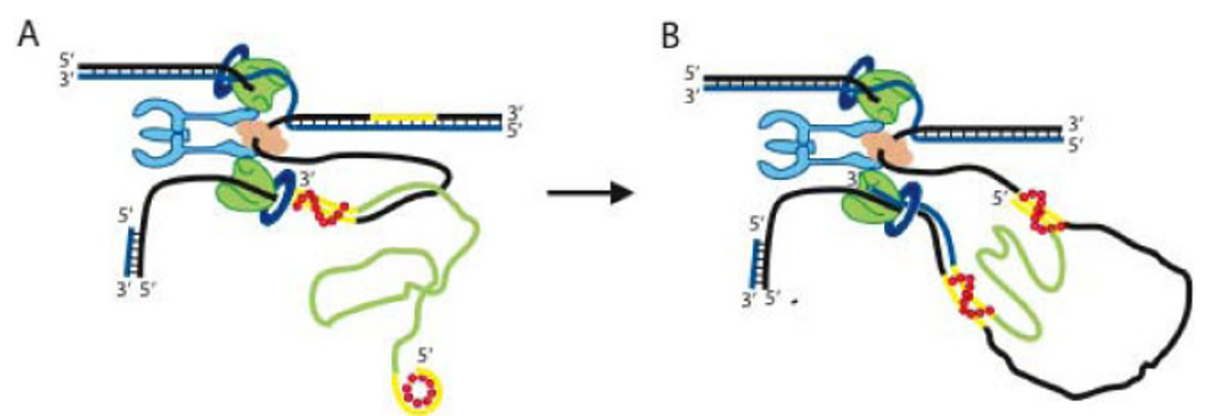

Figure 6 Model for a solo single-stranded DNA intermediate in Red recombination. Model for recombination at the replication fork. Annealing of an ssDNA molecule to complementary regions on the lagging strand at the replication fork is depicted. The ssDNA molecule comprises two flanking homology arms ( $\sim 50 \mathrm{nt}$; yellow), interspaced by a heterologous sequence (light green). The Red $\beta$ annealing intermediate is shown as a curved line of red dots. The leading strand is shown in blue, lagging strand in black, DnaB helicase in light orange, the two Pol III holoenzymes are green, which are tethered to the $\gamma / \tau$ clamp loader (light blue), and the $\beta$ sliding clamps are dark blue rings. (A) The Red $\beta$ ssDNA protein complex anneals the 3' end first, which then primes DNA synthesis for an Okazaki fragment. (B) After replication fork progression, the second homology region becomes exposed and annealing of the $5^{\prime}$ homology arm creates the ssDNA heteroduplex intermediate. 

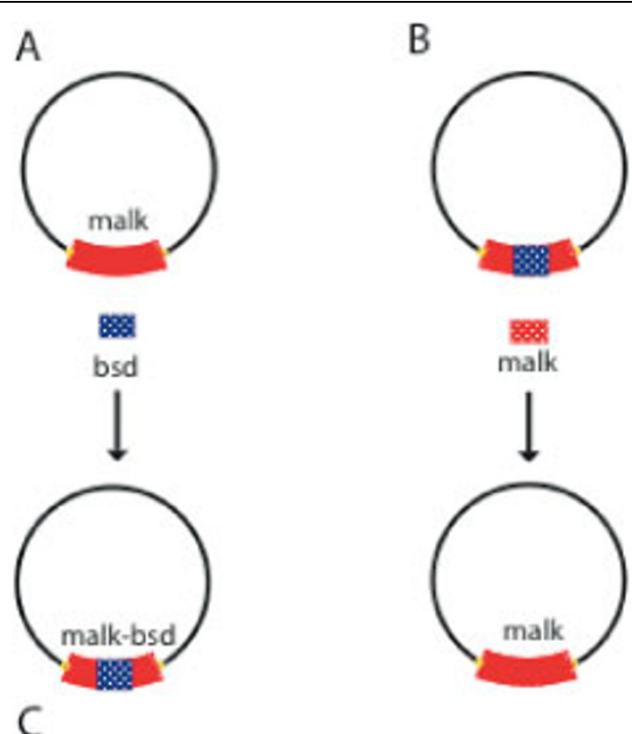

C

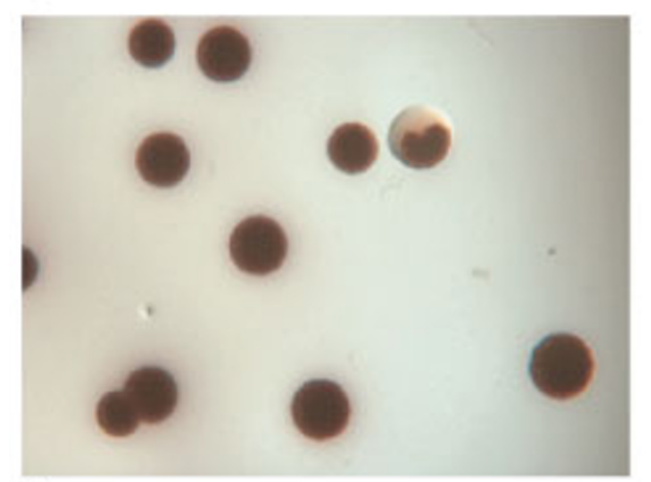

D

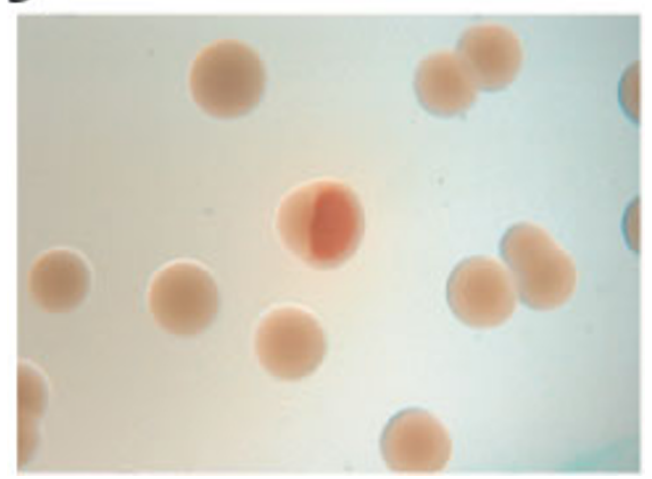

Figure 7 In situ recombination assay based on colony colour (A) Schematic illustration of the recombination assay, which leads to an insertion of the blasticidin resistance gene (bsd) into the genomic malK locus. Insertion mutates the malk gene, so recombinant colonies are white when plated on MacConkey agar. (B) Schematic representation of the reverse recombination event, which restores the functionality of the malk gene by deleting bsd, which was inserted in (A). The linear substrate was a $400 \mathrm{bp}$ dsDNA fragment of the malk gene. (C) Picture of red (non-recombinant) and half white/red (recombinant) colonies obtained using the design shown in (A). (D) Picture of white (non-recombinant) and half red/white (recombinant) colonies obtained using the recombination design shown in (B).
Table 1 Time course of recombination products at malK as scored by colony colour

\begin{tabular}{cccc}
\hline Time (min) before plating & \multicolumn{2}{c}{ Half white/ } & Full white no./7000 \\
red colonies & Percent & no./7000 & \\
\hline 5 & 93 & 13 & 1 \\
\hline 10 & 92 & 11 & 1 \\
\hline 15 & 86 & 12 & 2 \\
\hline 20 & 81 & 13 & 3 \\
\hline 25 & 76 & 13 & 4 \\
\hline 30 & 77 & 10 & 3 \\
\hline 60 & 40 & 6 & \\
\hline
\end{tabular}

Second we tested the impact of altering the length of the $3^{\prime}$ or $5^{\prime}$ homology arms of the LSP by making one homology arm shorter (30 rather than 50 nts; Figure $8 \mathrm{~B})$. As expected, recombination was most efficient when both homology arms were 50 nts (50 50). Reducing the $5^{\prime}$ end (30 50) dramatically reduced efficiency whereas reducing the 3 ' end (50 30) had a moderate effect. Notably reduction of both homology arms (30 30) was similar to the $5^{\prime}$ end reduction only, indicating that the length of the $5^{\prime}$ arm is the critical element.

These tests lend support to the beta recombination model by illustrating the non-equivalence of the two homology arms.

\section{The length of deletion has little effect but the length of insertion is critical}

The experiments performed above used small cassettes $(<1 \mathrm{~kb})$ for replacement targeting to achieve similarly sized deletions $(<1 \mathrm{~kb})$ in the target molecule. To determine whether larger deletions or insertions influence the mechanism, we built assays for the experiments of Figure 9. In particular we were interested to look at deletions across size ranges including $1 \mathrm{~kb}$, which is the average size of Okazaki fragments in E. coli, in case a discontinuity around $1 \mathrm{~kb}$ could be observed. For Figure 9A, we made a series of substrates by inserting from 4 to 4000 bps into the kanamycin resistance gene (neo) in a BAC. These insertions were then deleted by a short (100 bp) fragment to restore the neo gene. The $100 \mathrm{bp}$ fragment was generated in the four variations of $5^{\prime}$ phosphorylation and hydroxylation as illustrated. In a complementary assay to examine larger regions, one homology region was first inserted at various distances from the other around a BAC from 0.5 to $50 \mathrm{~kb}$ (Figure 9B). Then the region in between was deleted by replacement with the blasticidin gene using a $500 \mathrm{bp}$ fragment that was differentially 5 ' phosphorylated. Although the shorter deletions $(<500 \mathrm{bps})$ were most efficient, there was overall very little drop in efficiency with increasing size 
A

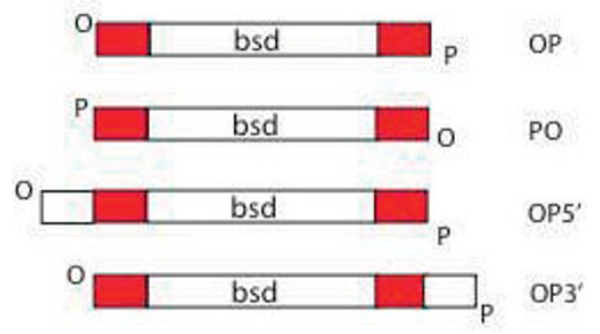

B

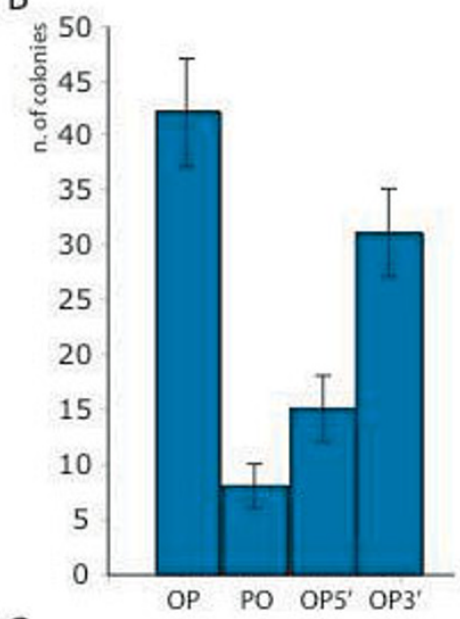

C

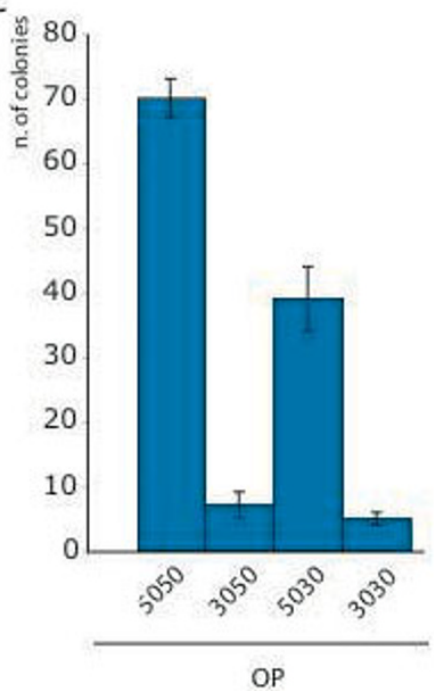

Figure 8 Terminal non-homologies indicate an underlying asymmetry. (A) The substrates are illustrated showing the location of the $50 \mathrm{bp}$ homology arms (red), hydroxylated (O) or phosphorylated (P) $5^{\prime}$ ends and the additional non-homology (30 bp) on one end or the other. OP5' refers to the addition of the non-homology onto the $5^{\prime}$ end of the LSP. OP3' refers to the addition of the non-homology on the $3^{\prime}$ end of the LSP. (B) Results using the substrates shown in (A) after recombination into a BAC in the presence of Red $\alpha \beta \gamma$. (C) The same assay as in (B) except that the homology arms of the PO substrate $(5050)$ were altered to be shorter on the $5^{\prime}$ end of the LSP (30 50), the $3^{\prime}$ end (50 30) or both (30 30). of deletion from 0.5 to $50 \mathrm{~kb}$. Furthermore, the strand preference for the LSP was maintained throughout all deletion sizes, even to $50 \mathrm{~kb}$.

In contrast to deletions, varying the insertion size revealed a vital insight. The efficiency of beta recombination, that is the preference for the LSP over its complementary strand, decreased with increasing size (Figure 9D). At $1 \mathrm{~kb}$, the LSP preference was large, whereas at $2 \mathrm{~kb}$ it was diminished and at $3 \mathrm{~kb}$ there appeared to be no preference for the LSP over the other ways to establish recombinants, including via the PP substrate, which is presumably symmetrically resected by Red $\alpha$. This relationship between length and efficiency of beta recombination could be due to limitations in the processivity or speed of Red $\alpha$ exonuclease activity $[21,42]$. Whatever the reason, this result not only indicates an important practical relationship between insertion size and beta recombination efficiency but also the possibility that dsDNA recombination mediated by the Red proteins can occur by another mechanism in addition to beta recombination.

\section{Ends-in recombination illustrates a second pathway for Red recombination}

To establish whether Red dsDNA recombination can operate through another mechanism(s), we compared replacement recombination, also known as 'ends-out', with 'ends-in' recombination using the same target and homology regions (Figure 9D). As described above, replacement recombination showed the expected preference for the LSP. However insertional recombination did not show a strand preference. Notably removal of RuvABC had no impact on either beta recombination or the alternative pathway. Furthermore the beta recombination pathway is clearly more efficient than the alternatives at insert sizes of less than $3 \mathrm{~kb}$.

\section{Discussion}

We present a new model for replacement targeting by the lambda Red pathway based on a heteroduplex intermediate that incorporates only one strand of the dsDNA substrate, which we term 'beta' recombination (Figure 6). In contrast to a double crossover model, beta recombination does not involve symmetrical resection of the dsDNA substrate from each end by the $5^{\prime}$ to $3^{\prime}$ Red $\alpha$ exonuclease. Instead one strand is removed completely whilst the other strand remains unresected and contains both homology arms. Like oligonucleotide-directed mutagenesis mediated by $\operatorname{Red} \beta$, the strand that delivers more recombinants is the strand whose 3 ' end can serve as a primer for lagging strand synthesis (termed the LSP). Hence we suggest that this mode of dsDNA recombination, like ssDNA recombination, also occurs at the replication fork. 

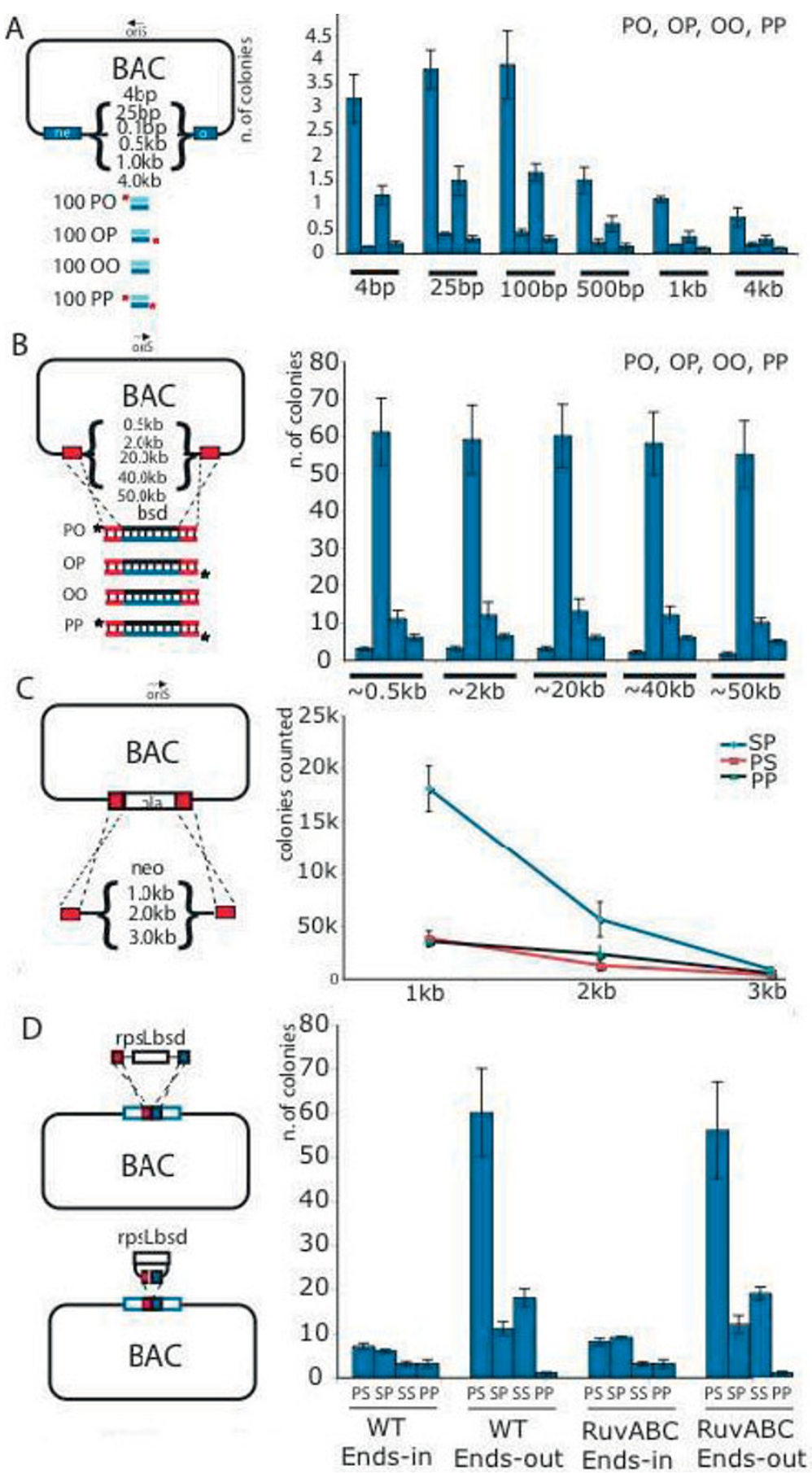

Figure 9 Increasing the deletion size has little effect but increasing the substrate size indicates limits to beta recombination. (A) Schematic illustration of an assay using a BAC carrying variously sized insertions in the neo gene, from 4 to 4000 bps as indicated, which were deleted by a $100 \mathrm{bp}$ fragment to restore kanamycin resistance. Results are plotted at the right. (B) Schematic illustration of an assay using a BAC assay with one homology arm (red) placed at various distances from 0.5 to $50 \mathrm{~kb}$ to the other homology arm (red) as indicated. The region between these two sequences was deleted by insertion of a 500 bp fragment carrying blasticidin resistance as plotted to the right. (C) Schematic illustration of an assay to insert either 1,2 or $3 \mathrm{~kb}$ fragments carrying the neo gene into the same place on a BAC. The fragments were synthesized to have 5' phosphorylated (P) or phosphothioated (S) ends as indicated in the plotted curves to the right. (D) Schematic illustration of an assay showing the insertion of a $1 \mathrm{~kb}$ fragment carrying the rps $L$ and blasticidin (bsd) genes into the same site on a BAC. The $1 \mathrm{~kb}$ fragment was identical except that the homology arms were arranged for ends-out (replacement, above) or ends-in (below) recombination, and synthesized with combinations of $5^{\prime}$ phoshorylated $(\mathrm{P})$ or phosphothioated $(\mathrm{S})$ ends as indicated in the plot to the right. The experiments were performed in our standard host (GB2005 $=$ WT) or the same host after deletion of ruvabc. 
The reliance of Red recombination on replication was identified a long time ago [49]. However this relationship was subsequently attributed to the replicationdependent generation of recombinogenic dsDNA ends [50] rather than a direct involvement of the replication fork. Furthermore, support for a replication-independent, annealing mechanism was found [28]. Also, the relationship between Red recombination and replication has been complicated by the influence of RecA [36,51-53] presumably because RecA can assist Red recombination by mediating replication-independent strand invasion. In the presence of replication, RecA can make a substantial contribution to Red mediated recombination under certain circumstances [51] but not others $[32,35,54,55]$. The discovery, that the Red proteins and the closely related RecE/RecT proteins can mediate an apparent strand invasion activity in the absence of RecA $[29,30]$ led to a new linkage between Red recombination and replication. Because Red-mediated oligonucleotidedirected mutagenesis showed strand preference according to the direction of replication, annealing of ssDNA primers at the replication fork for lagging strand synthesis was proposed $[7,31,32]$. Using an in vivo assay based on phage infection and restriction cleavage, it has been recently shown that Red-mediated dsDNA recombination requires replication [39]. Here we also show that replication of the target molecule is required for Red-mediated dsDNA recombination (Figure 2).

The beta recombination model (Figure 6) implies that the two homology arms of the dsDNA substrate are not equivalent. The homology arm at the 3 ' end of the LSP is more likely to prime recombination than the other $3^{\prime}$ homology arm. Also, the homology arm at the 3' end of the LSP should anneal before the homology arm at the 5 ' end of the LSP because its' complementary sequence is exposed earlier at the replication fork. Hence $\operatorname{Red} \beta$ likely stabilizes annealing of the 3' homology arm while the replication fork continues. Later, when the region complementary to the $5^{\prime}$ homology arm is exposed, the 5 ' homology arm anneals and the heteroduplex that promotes recombination is established. On this point, it was recently shown that the replication fork continues even though cleavage has occurred in the recently replicated DNA [56]. Therefore it appears that the progress of the replication fork in $E$. coli is not sensitive to events behind it. Support for the idea that the two homology arms in a dsDNA substrate are not equivalent for Red recombination has been recently published [57]. These authors showed recombination asymmetries that can be explained by the beta recombination model. Here we show that heterologous sequence at the 3 ' end of the LSP has little deleterious impact, presumably because it is rapidly cut away in the process of priming replication, whereas heterologous sequence at the 5 ' end of the LSP interferes with recombination. Also we suggest that the 3 ' annealed end is rapidly stabilized by priming DNA synthesis, which is supported by observation that the length of the $5^{\prime}$ homology arm is more important than the length of the 3 ' homology arm (Figure 8 ). These asymmetric effects further support the beta recombination model and have practical implications for recombineering efficiency.

To test the idea that beta recombination involves the proposed ssDNA heteroduplex, we developed an in situ assay to see if replication of the heteroduplex generated one wild type and one recombinant daughter, which should be visible as colony sectoring (Figure 7). Colony sectoring was observed however this result could also be generated if the host cell harboured two copies of the target malK locus. Therefore we looked at colony sectoring as a time course of recombination before plating. At the shortest time points, virtually all recombinant colonies were two-coloured, whereas increasing the incubation time resulted in a greater proportion of onecoloured recombinant colonies indicating that cell division occurred after recombination and before plating. It also indicates that the majority of recombinant cells carried only one copy of the malk locus at the moment of recombination. Taken together with the evidence that Red recombination requires replication of the target, the colony sectoring results support the existence of a heteroduplex intermediate.

We, and others, have previously reported that large deletions can be readily achieved with Red recombination at efficiencies similar to small deletions. However this parameter has not been examined systematically. Here we looked carefully at deletions around the size of an Okazaki fragment $(\sim 1 \mathrm{~kb})$ in two different assays but did not see any discontinuity or any substantial loss of efficiency up to deletions of $50 \mathrm{~kb}$. Taken together with evidence supporting the subsequent replication of the heteroduplex after establishment (Figure 7), it appears that the $\operatorname{Red} \beta$ annealed complex is highly stable in vivo. This concords with the high stability of the complex in vitro $[5,22,23]$. Alternatively, a transient $\operatorname{Red} \beta$ annealed complex could be rapidly stabilized once it has served to prime replication.

Although we present evidence that Red $\beta$ recombination with dsDNA is mediated via solo ssDNA intermediates, our evidence does not exclude additional mechanisms. Indeed, we found circumstances where less efficient levels of recombination appear to be evidence of another mechanism (Figure 9), which could be explained by a template-switching model [39]. The Red proteins can also mediate recombination by a simple annealing mechanism [28]. Furthermore the apparent recombination on the leading strand (that is, from the strand complementary to the LSP) requires an explanation that could also be 
mechanistically quite different. Consequently it seems that the Red proteins, and $\operatorname{Red} \beta$ in particular, have the capacity to facilitate more than one recombination pathway. Notably, the role of the Red proteins in the lambda phage lifecycle remains unresolved [12,39], although an evolutionary role has been proposed [58]. Our evidence further strengthens the suggestion that the Red proteins promote the switch from theta to rolling-circle replication in the lambda lifecycle, possibly by more than one mechanism.

The beta recombination model also has some practical implications. As with oligonucleotide-directed mutagenesis, knowledge of the direction of replication can be used to optimize recombineering efficiencies. For many applications, the additional efficiency achieved is not necessary, particularly because the OO, SS and even PP configurations usually deliver more than enough product for DNA engineering exercises. However in certain cases, such as high throughput recombineering $[59,60]$, the further gain of efficiency can deliver useful reliability or help achieve complex tasks (unpublished observations). Furthermore we suggest that the beta model may have relevance for applied HR in other systems. Because strand bias in oligonucleotide-directed mutagenesis has also been observed in yeast [61], it will be interesting to test whether asymmetrical dsDNA substrates show a similar efficiency profile. Exploring these parameters in mammalian cells could also be fruitful.

Several other practical points emerged; (i) two consecutive phosphothioates increased recombination efficiency, presumably because they stabilized the 5 ' end against exonucleases whilst being permissive for the further steps of recombination. Notably the dsDNA substrates with phosphothioates at both $5^{\prime}$ ends were the second-most efficient configuration in our experiments. We suggest that these substrates are also utilized by beta recombination, however are made single-stranded by a helicase activity; (ii) identical DNA fragments produced by PCR or restriction digestion usually differ with respect to phosphorylation at their $5^{\prime}$ ends. This can lead to different recombination efficiencies; (iii) heterologous extensions beyond the homology arms are better tolerated at 3' but not 5' LSP ends; (iv) shorter cassettes are significantly more efficiently incorporated than longer, which has implications for high throughput and complex recombineering tasks.

\section{Conclusions}

We present evidence supporting a model for homologous recombination mediated by $\operatorname{Red} \beta$ acting via a single stranded heteroduplex intermediate formed at the replication fork. Although other mechanisms are not excluded, it appears that the remarkable efficiency of Red recombination utilizes this single stranded intermediate. The model has several practical implications for recombineering when highly efficient applications are required.

\section{Methods \\ Strains}

All the strains and substrates used in this work were generated by recombineering. GB2005 is a derivative of HS996. The Rac prophage recE and recT, and DLP12 prophage $y b c C$ that encodes a putative exonuclease similar to the Red $\alpha$, were deleted to generate GB2005. Both deletions were done by insertion of a selectable marker (kan) flanked by lox66 and lox71 sites; subsequently the selectable marker was removed by Cre recombination. The $m u t S$ and $s b c B$ deletion strains were generated in a similar way by removing the whole open reading frame of the respective genes, however the cassette used for deletion was the puromycin resistant gene (puro) flanked by rox sites. The puro cassette was subsequently excised by Dre recombination [62]. GB2005-pir was obtained by inserting a cassette containing the pir gene plus a selectable marker ( $k a n)$ flanked by lox71 and lox66 sites in the $y b c C$ locus. And then the selectable marker was removed by Cre recombination.

\section{Recombineering}

All recombination reactions were performed as follows unless stated. Cells carrying a pSC101 vector, either BAD-Red $\alpha \beta \gamma$ or BAD-Red $\beta$ were passaged by inoculation of $0.5 \mathrm{ml}$ from a fresh overnight culture into $25 \mathrm{ml}$ $\mathrm{LB}, 5 \mu \mathrm{g} / \mathrm{ml}$ tetracycline and grown at $30^{\circ} \mathrm{C}$ for 2 hours. Then protein expression was induced by addition of Larabinose to $0.2 \%$ and grown at $37^{\circ} \mathrm{C}$ for 45 minutes followed by centrifugation at $0^{\circ} \mathrm{C}$, resuspension in ice cold $10 \%$ glycerol and transfer to an ice cold Eppendorf tube. The cells were then washed 2 times with ice-cold $1 \mathrm{ml}$ of $10 \%$ glycerol in the Eppendorf tube and then diluted to $600 \mu \mathrm{l}$ ice cold $10 \%$ glycerol. $30 \mu \mathrm{l}$ were used for each electroporation in pre-chilled $1 \mathrm{~mm}$ Eppendorf 100 $\mu \mathrm{l}$ cuvettes using an Eppendorf 2510 set at $1350 \mathrm{~V}$. After electroporation, the cells were resuspended in Lbroth and incubated at $37^{\circ} \mathrm{C}$ for 45 minutes before plating. All experiments were repeated three times using 50 ng of co-electroporated plasmid as an internal control for electroporation efficiency.

\section{Oligonucleotide sequences}

The sequences of the oligonucleotides used for recombineering can be found in Additional file 1.

\section{Replication assays}

The replication origin in the $\mathrm{R} 6 \mathrm{~K}$-amp plasmid was exchanged using a PCR product containing the blasticidin resistance gene (bsd) and the pBR322 origin of replication. A plasmid was built to serve as template for the 
PCR reaction and homology arms to the R6K plasmid were added by the PCR reaction. Then, co-electroporation of $200 \mathrm{ng}$ of the R6K-amp plasmid DNA with 200 ng of PCR product into cells expressing Red $\alpha \beta \gamma$ under arabinose induction in presence (GB2005-pir) or absence (GB2005) of the Pir protein was followed by plating onto blasticidin $(50 \mu \mathrm{g} / \mathrm{ml})$ plates. The restoration of a functional pir gene in a $\mathrm{R} 6 \mathrm{~K} \gamma$ ori plasmid was done by recombining a cassette containing the ampicillin resistance gene $(a m p)$ plus the 3' end of the pir gene into a R6K-cm-pir* plasmid that carried only the $5^{\prime}$ end of the pir gene. The PCR template was pR6K-amp-pir*. Both pR6K-cm-pir" and pR6K-amp-pir* were constructed from pR6K-hyg-pir. The recombinants were identified and counted on ampicillin plates $(100 \mu \mathrm{g} / \mathrm{ml})$.

\section{$\lambda$ exonuclease (Red $\alpha$ ) digestion}

was performed to isolate ssDNA from asymmetrically phosphorylated dsDNA. 100 pmol of dsDNA were digested with 1 unit of $\lambda$ exonuclease (New England Biolabs) for 1 hour at $37^{\circ} \mathrm{C}$, followed by purification using gel extraction kit (QIAGEN, Germany). 2.5 volumes of denaturating buffer ( $95 \%$ formamide, $10 \mathrm{mM}$ $\mathrm{NaOH}, 0.05 \%$ xylene cyanol, $0.05 \%$ bromophenol blue) was added to 1 volume of digestion product before boiling for 5 minutes at $95^{\circ} \mathrm{C}$ and placing on ice. $20 \mu \mathrm{l}$ were loaded per each lane containing $10 \mu \mathrm{l}$ from the boiled sample and $10 \mu \mathrm{l}$ from the unboiled sample. Native polyacrylamide gel electrophoresis was used for SSCP overnight (14 hours) at $50 \mathrm{~V}$. ssDNA recombination was performed by electroporating 2 pmoles of ssDNA generated from the in vitro Red $\alpha$ digestion. For the ssOR experiment 1 pmole HPLC-purified oligonucletide was used.

The samples for the in vivo digestion experiment were prepared using 5 vials of competent cells (each $40 \mu \mathrm{l}$ ), which were each transformed with $170 \mathrm{ng}$ of asymmetrically phopshorylated bsd cassette. $1 \mathrm{ml}$ of L-broth was added to the cells and they were incubated 5 minutes at $37^{\circ} \mathrm{C}$. After extracting the DNA using a standard plasmid mini-prep, the 5 DNAs were pooled and loaded as above on an SSCP gel.

\section{Differentially phosphorylated cassettes}

were generated by PCR using differentially end-modified oligonucleotides. Four different kinds of modification were exploited: normal unmodified 5 ' ends (O oligonucleotides), phosphorylated 5 ' ends (P oligonucleotides), insertion of 2 phosphothioate linkages between the first and the second and the second and the third nucleotides starting from 5 ' end (S oligonucleotides) and both phosphorylated 5 ' ends combined with 2 phosphothioate linkages (PS or SP oligonucleotides).

\section{Recombinations with differentially 5 ' end phosphorylated dsDNA}

were done by electroporating 2 pmoles and plating $50 \mu \mathrm{l}$ of the $1 \mathrm{ml}$ resuspension. The pBelo-BAC-bla and pBeloBAC-inv-bla were generated by inserting the ampicillin resistance gene $(\mathrm{amp})$ into the neo gene of pBelo-BAC11neo* in both orientations. The same cassette was also inserted in both orientations in the ara-leu locus on the chromosome. The BACs used for deletion experiments were generating by inserting fragments of different sizes into the NcoI site of the neo gene. The $4 \mathrm{bp}$ insertion is the same neo* used previously [32]. A 100 bp long cassette having homology to both sides of the insertion was used to restore a functional neo gene. The bigger deletion series used a mouse pax 8 BAC containing a cassette (neo) inserted at different distances from a chosen point $(0.5,2,20,40,50 \mathrm{~kb})$. The same rpsL-bsd cassette was used for the amplification in the ends-in and ends-out experiment.

\section{Colony colour test}

Part of the malK gene (300 bps) was deleted by replacement with the blasticidin resistance gene. This was restored with a cassette containing the missing $300 \mathrm{bp}$ plus $50 \mathrm{bp}$ of further homology to malK either side. To obtain a high efficiency of recombination $(\sim 1 / 500$ colonies), $1 \mu \mathrm{g}$ of a PS cassette was electroporated and 100 $\mu \mathrm{l}$ of $10^{6}$ dilution were plated on MacConkey agar plus maltose. The experiment was performed at $30^{\circ} \mathrm{C}$ to minimize the number of replication forks. The restoration of a functional malK gave higher efficiency of recombination (probably due to the shorter length of the transformed cassette (400 bp) and was used for the experiment in Table1.

\section{Additional material}

Additional file 1: Supplementary information. Oligonucleotides used

\section{Acknowledgements}

We thank Andy Porter for critical comments on the manuscript and David Sherratt for discussions. Funding was provided by the National Genome Forschung Netzwerk program, DiGtoP (Disease Genes to Pathways) and the Saxonian Ministry for Science and Arts, EFRE program for the Biotechnology Center, Technische Universitaet Dresden.

Author details

${ }^{1}$ Genomics, Technische Universität Dresden, BiolnnovationsZentrum, Tatzberg 47-51, D-01307 Dresden, Germany. ${ }^{2}$ Gene Bridges GmbH,

BiolnnovationsZentrum, Tatzberg 47-51, D-01307 Dresden, Germany.

\section{Authors' contributions}

$M M, A E, J F$ and $A F$ performed the experiments. $Y Z$ made the initial discovery. MM, AE, JF, YZ and AFS designed the experiments. AFS wrote the paper. All authors approved the final version. 
Received: 3 March 2010 Accepted: 29 July 2010 Published: 29 July 2010

\section{References}

1. Cox MM, Goodman MF, Kreuzer KN, Sherratt DJ, Sandler SJ, Marians KJ: The importance of repairing stalled replication forks. Nature 2000, 404:37-41.

2. Kuzminov A: DNA replication meets genetic exchange: chromosomal damage and its repair by homologous recombination. Proc Natl Acad Sci USA 2001, 98:8461-8.

3. Kuzminov A: Recombinational repair of DNA damage in Escherichia coli and bacteriophage lambda. Microbiol Mol Biol Rev 1999, 63:751-813, table of contents.

4. Iyer $L M$, Koonin EV, Aravind L: Classification and evolutionary history of the single-strand annealing proteins, RecT, Redbeta, ERF and RAD52. BMC Genomics 2002, 3:8.

5. Erler A, Wegmann S, Elie-Caille $C$, Bradshaw CR, Maresca M, Seidel R, Habermann B, Muller DJ, Stewart AF: Conformational Adaptability of Redbeta during DNA Annealing and Implications for its Structural Relationship with Rad52. J Mol Biol 2009, 391(3):586-98, (2009).

6. Capecchi MR: Altering the genome by homologous recombination. Science 1989, 244:1288-92.

7. Court DL, Sawitzke JA, Thomason LC: Genetic engineering using homologous recombination. Annu Rev Genet 2002, 36:361-88.

8. Glaser S, Anastassiadis K, Stewart AF: Current issues in mouse genome engineering. Nat Genet 2005, 37:1187-93.

9. van der Weyden L, Adams DJ, Bradley A: Tools for targeted manipulation of the mouse genome. Physiol Genomics 2002, 11:133-64.

10. Copeland NG, Jenkins NA, Court DL: Recombineering: a powerful new tool for mouse functional genomics. Nat Rev Genet 2001, 2:769-79.

11. Muyrers JP, Zhang Y, Stewart AF: Techniques: Recombinogenic engineering-new options for cloning and manipulating DNA. Trends Biochem Sci 2001, 26:325-31.

12. Poteete AR: What makes the bacteriophage lambda Red system useful for genetic engineering: molecular mechanism and biological function. FEMS Microbiol Lett 2001, 201:9-14.

13. Langston LD, Symington LS: Gene targeting in yeast is initiated by two independent strand invasions. Proc Natl Acad Sci USA 2004, 101:15392-7.

14. $L i J$, Read $L R$, Baker MD: The mechanism of mammalian gene replacement is consistent with the formation of long regions of heteroduplex DNA associated with two crossing-over events. Mol Cell Biol 2001, 21:501-10.

15. Paques $F$, Haber JE: Multiple pathways of recombination induced by double-strand breaks in Saccharomyces cerevisiae. Microbiol Mol Biol Rev 1999, 63:349-404.

16. Smith GR: Homologous recombination near and far from DNA breaks: alternative roles and contrasting views. Annu Rev Genet 2001, 35:243-74.

17. Morrow DM, Connelly C, Hieter P: "Break copy" duplication: a model for chromosome fragment formation in Saccharomyces cerevisiae. Genetics 1997, 147:371-82.

18. Leung W, Malkova A, Haber JE: Gene targeting by linear duplex DNA frequently occurs by assimilation of a single strand that is subject to preferential mismatch correction. Proc Natl Acad Sci USA 1997, 94:6851-6.

19. Kovall R, Matthews BW: Toroidal structure of lambda-exonuclease. Science 1997, 277:1824-7.

20. Perkins TT, Dalal RV, Mitsis PG, Block SM: Sequence-dependent pausing of single lambda exonuclease molecules. Science 2003, 301:1914-8.

21. Subramanian K, Rutvisuttinunt W, Scott W, Myers RS: The enzymatic basis of processivity in lambda exonuclease. Nucleic Acids Res 2003, 31:1585-96.

22. Karakousis G, Ye N, Li Z, Chiu SK, Reddy G, Radding CM: The beta protein of phage lambda binds preferentially to an intermediate in DNA renaturation. J Mol Biol 1998, 276:721-31.

23. Wu Z, Xing X, Bohl CE, Wisler JW, Dalton JT, Bell CE: Domain structure and DNA binding regions of beta protein from bacteriophage lambda. J Biol Chem 2006, 281:25205-14.

24. Court R, Cook N, Saikrishnan K, Wigley D: The crystal structure of lambdaGam protein suggests a model for ReCBCD inhibition. J Mol Biol 2007, 371:25-33.

25. Murphy KC: The lambda Gam protein inhibits RecBCD binding to dsDNA ends. J Mol Biol 2007, 371:19-24.

26. Poteete $A R$, Fenton $A C$, Nadkarni A: Chromosomal duplications and cointegrates generated by the bacteriophage lamdba Red system in Escherichia coli K-12. BMC Mol Biol 2004, 5:22.
27. Rybalchenko N, Golub El, Bi B, Radding CM: Strand invasion promoted by recombination protein beta of coliphage lambda. Proc Natl Acad Sci USA 2004, 101:17056-60.

28. Stahl MM, Thomason L, Poteete AR, Tarkowski T, Kuzminov A, Stahl FW: Annealing vs. invasion in phage lambda recombination. Genetics 1997, 147:961-77.

29. Muyrers JP, Zhang Y, Testa G, Stewart AF: Rapid modification of bacterial artificial chromosomes by ET-recombination. Nucleic Acids Res 1999, 27:1555-7.

30. Zhang Y, Buchholz F, Muyrers JP, Stewart AF: A new logic for DNA engineering using recombination in Escherichia coli. Nat Genet 1998, 20:123-8.

31. Ellis HM, Yu D, DiTizio T, Court DL: High efficiency mutagenesis repair, and engineering of chromosomal DNA using single-stranded oligonucleotides. Proc Natl Acad Sci USA 2001, 98:6742-6.

32. Zhang Y, Muyrers JP, Rientjes J, Stewart AF: Phage annealing proteins promote oligonucleotide-directed mutagenesis in Escherichia coli and mouse ES cells. BMC Mol Biol 2003, 4:1.

33. Costantino N, Court DL: Enhanced levels of lambda Red-mediated recombinants in mismatch repair mutants. Proc Natl Acad Sci USA 2003, 100:15748-53.

34. Li XT, Costantino N, Lu LY, Liu DP, Watt RM, Cheah KS, Court DL, Huang JD: Identification of factors influencing strand bias in oligonucleotidemediated recombination in Escherichia coli. Nucleic Acids Res 2003, 31:6674-87.

35. Muyrers JP, Zhang Y, Buchholz F, Stewart AF: RecE/RecT and Redalpha/ Redbeta initiate double-stranded break repair by specifically interacting with their respective partners. Genes Dev 2000, 14:1971-82.

36. Poteete AR, Fenton AC: Genetic requirements of phage lambda redmediated gene replacement in Escherichia coli K-12. J Bacteriol 2000, 182:2336-40.

37. Briggs GS, Mahdi AA, Weller GR, Wen Q, Lloyd RG: Interplay between DNA replication recombination and repair based on the structure of RecG helicase. Philos Trans R Soc Lond B Biol Sci 2004, 359:49-59.

38. Poteete $A R$, Fenton $A C$, Murphy KC: Roles of RuvC and RecG in phage lambda red-mediated recombination. J Bacteriol 1999, 181:5402-8.

39. Poteete AR: Involvement of DNA replication in phage lambda Redmediated homologous recombination. Mol Microbiol 2008, 68:66-74

40. Filutowicz M, McEachern MJ, Helinski DR: Positive and negative roles of an initiator protein at an origin of replication. Proc Natl Acad Sci USA 1986, 83:9645-9.

41. Stalker DM, Filutowicz M, Helinski DR: Release of initiation control by a mutational alteration in the R6K pi protein required for plasmid DNA replication. Proc Natl Acad Sci USA 1983, 80:5500-4.

42. Little JW: An exonuclease induced by bacteriophage lambda. II. Nature of the enzymatic reaction. J Biol Chem 1967, 242:679-86.

43. Orita M, Iwahana $H$, Kanazawa $H$, Hayashi K, Sekiya T: Detection of polymorphisms of human DNA by gel electrophoresis as single-strand conformation polymorphisms. Proc Natl Acad Sci USA 1989, 86:2766-70.

44. Sunnucks P, Wilson AC, Beheregaray LB, Zenger K, French J, Taylor AC: SSCP is not so difficult: the application and utility of single-stranded conformation polymorphism in evolutionary biology and molecular ecology. Mol Ecol 2000, 9:1699-710.

45. Yu D, Sawitzke JA, Ellis H, Court DL: Recombineering with overlapping single-stranded DNA oligonucleotides: testing a recombination intermediate. Proc Natl Acad Sci USA 2003, 100:7207-12.

46. Busam RD: Structure of Escherichia coli exonuclease I in complex with thymidine 5'-monophosphate. Acta Crystallogr D Biol Crystallogr 2008, 64:206-10.

47. Prasher D, Kasunic DA, Kushner SR: Physical and genetic characterization of the cloned sbcB (exonuclease I) region of the Escherichia coli genome. J Bacteriol 1983, 153:903-8.

48. Shuman HA, Silhavy TJ: Identification of the malK gene product. A peripheral membrane component of the Escherichia coli maltose transport system. J Biol Chem 1981, 256:560-2.

49. Stahl FW, McMilin KD, Stahl MM, Crasemann JM, Lam S: The distribution of crossovers along unreplicated lambda bacteriophage chromosomes. Genetics 1974, 77:395-408.

50. Thaler DS, Stahl MM, Stahl FW: Double-chain-cut sites are recombination hotspots in the Red pathway of phage lambda. J Mol Biol 1987, 195:75-87. 
51. Murphy KC: Use of bacteriophage lambda recombination functions to promote gene replacement in Escherichia coli. J Bacterio/ 1998, 180:2063-71.

52. Nussbaum A, Shalit M, Cohen A: Restriction-stimulated homologous recombination of plasmids by the RecE pathway of Escherichia coli. Genetics 1992, 130:37-49.

53. Poteete AR, Fenton AC: Efficient double-strand break-stimulated recombination promoted by the general recombination systems of phages lambda and P22. Genetics 1993, 134:1013-21.

54. Wang J, Sarov M, Rientjes J, Fu J, Hollak H, Kranz H, Xie W, Stewart AF, Zhang Y: An improved recombineering approach by adding RecA to lambda Red recombination. Mol Biotechnol 2006, 32:43-53.

55. Yu D, Ellis HM, Lee EC, Jenkins NA, Copeland NG, Court DL: An efficient recombination system for chromosome engineering in Escherichia coli. Proc Natl Acad Sci USA 2000, 97:5978-83.

56. Eykelenboom JK, Blackwood JK, Okely E, Leach DR: SbcCD causes a double-strand break at a DNA palindrome in the Escherichia coli chromosome. Mol Cell 2008, 29:644-51.

57. Lim SI, Min BE, Jung GY: Lagging strand-biased initiation of red recombination by linear double-stranded DNAs. J Mol Biol 2008, 384:1098-105

58. Martinsohn JT, Radman M, Petit MA: The lambda red proteins promote efficient recombination between diverged sequences: implications for bacteriophage genome mosaicism. PLoS Genet 2008, 4:e1000065.

59. Poser I, Sarov M, Hutchins JR, Heriche JK, Toyoda Y, Pozniakovsky A, Weigl D, Nitzsche A, Hegemann B, Bird AW, Pelletier L, Kittler R, Hua S, Naumann R, Augsburg M, Sykora MM, Hofemeister H, Zhang $Y$, Nasmyth $K$, White KP, Dietzel S, Mechtler K, Durbin R, Stewart AF, Peters JM, Buchholz F, Hyman AA: BAC TransgeneOmics: a high-throughput method for exploration of protein function in mammals. Nat Methods 2008, 5:409-15.

60. Sarov M, Schneider S, Pozniakovski A, Roguev A, Ernst S, Zhang Y Hyman AA, Stewart AF: A recombineering pipeline for functional genomics applied to Caenorhabditis elegans. Nat Methods 2006, 3:839-44.

61. Storici F, Lewis LK, Resnick MA: In vivo site-directed mutagenesis using oligonucleotides. Nat Biotechnol 2001, 19:773-6.

62. Anastassiadis K, Fu J, Patsch C, Hu S, Weidlich S, Duerschke K, Buchholz F, Edenhofer F, Stewart AF: Dre recombinase Cre like is a highly efficient site specific recombinase in $\mathrm{E}$. coli, mammalian cells and mice. Disease Models and Mechanisms 2009, 2(9-10):508-15.

doi:10.1186/1471-2199-11-54

Cite this article as: Maresca et al.: Single-stranded heteroduplex intermediates in $\lambda$ Red homologous recombination. BMC Molecular Biology 2010 11:54.

\section{Submit your next manuscript to BioMed Central and take full advantage of:}

- Convenient online submission

- Thorough peer review

- No space constraints or color figure charges

- Immediate publication on acceptance

- Inclusion in PubMed, CAS, Scopus and Google Scholar

- Research which is freely available for redistribution

Submit your manuscript at www.biomedcentral com/submit
C Biomed Central 